Brief note

\title{
EFFECTIVE INTERFACIAL TENSION EFFECT ON THE INSTABILITY OF STREAMING RIVLIN-ERICKSEN ELASTICO-VISCOUS FLUID FLOW THROUGH A POROUS MEDIUM
}

\author{
M. SINGH \\ Department of Mathematics \\ Govt. Post Graduate College Seema (Rohru) \\ Distt Shimla (H.P), INDIA \\ E-mail: mahinder_singh91@rediffmail.in; drmsmath78@gmail.com
}

\begin{abstract}
The instability of the plane interface between two uniform, superposed and streaming Rivlin-Ericksen elastico-viscous fluids through porous media, including the 'effective interfacial tension' effect, is considered. In the absence of the 'effective interfacial tension' stability/instability of the system as well as perturbations transverse to the direction of streaming are found to be unaffected by the presence of streaming if perturbations in the direction of streaming are ignored, whereas for perturbation in all other directions, there exists instability for a certain wave number range. The 'effective interfacial tension' is able to suppress this Kelvin-Helmholtz instability for small wavelength perturbations, the medium porosity reduces the stability range given in terms of a difference in streaming velocities.
\end{abstract}

Key words: Kelvin-Helmholtz instability, Rivlin-Ericksen elastico-viscous fluids in porous medium.

\section{Introduction}

A detailed account of the Kelvin-Helmholtz instability of Newtonian fluids has been given by Chandrasekhar [1]. With the growing importance of non-Newtonian fluids in modern technology and industries, the investigations on such fluids are desirable. One important class of elastico-viscous fluids (a sub-class of non-Newtonian fluids) is the Rivlin-Ericksen elastico-viscous fluid, the constitutive equation for which has been proposed by Rivlin and Ericksen [2].

In recent years, the investigation of flow of fluids through porous media has become an important topic due to the recovery of crude oil from the pores of reservoir rock. A great number of applications in geophysics may be found in a book by Phillips [3]. When the fluid permeates a porous material, the gross effect is represented by Darcy's law. As a result of this macroscopic law, heuristically, the usual viscous term in the equation of Rivlin-Ericksen fluid motion is replaced by the resistance term $\left[-\frac{1}{k_{l}}\left(\mu+\mu^{\prime} \frac{\partial}{\partial t}\right) \boldsymbol{q}\right]$, where $\mu$ and $\mu^{\prime}$ are the viscosity and viscoelasticity of the Rivlin-Ericksen fluid, $k_{l}$ is the medium permeability and $\boldsymbol{q}$ is the Darcian (filter) velocity of the fluid.

A theoretical and experimental investigation of the instability of slow, immiscible, viscous liquidliquid displacement in porous media has been made by Chuoke et al. [4]. In flows through porous media, the front is not sharp (as in ordinary fluid dynamics) but is dispersed and broad. Chuoke et al. [4] assumed a macroscopic interface and an 'effective interfacial tension'. The instability of the plane interface between two uniform superposed and streaming fluids through a porous medium was investigated by Sharma and Spanos [5]. 
Keeping in mind the importance of non-Newtonian fluids in modern technology and industries, the present paper attempts to study the instability of streaming Rivlin-Ericksen elastico-viscous fluids in a porous medium.

\section{Formulation of the problem and perturbation equations}

The initial stationary state, whose stability we wish to examine, is that of an incompressible elasticoviscous fluid in a porous medium in which there is a horizontal streaming $\boldsymbol{U}(U(z), 0,0)$. The character of the equilibrium of this initial state is determined by supposing that the system is slightly disturbed and then following its further evolution.

In flows through porous media there are no sharp fronts and so no actual interfacial tensions at some prescribed levels $z_{s}$, as in ordinary fluid dynamics. However, there is a macroscopic interface (broad front) if viewed from a large distance, and by analogy with Leplace's formula, at each point of the macroscopic interface

$$
\left(p_{1}-p_{2}\right)_{z=z_{s}}=-T_{s}\left(c_{1}+c_{2}\right)
$$

where $c_{1}, c_{2}$ are the signed principal curvatures of the macroscopic interface and $T_{s}$ is the 'effective interfacial tension'. This is the first approximation to the problem in a porous medium and this theory was used and enunciated by Chuoke et al. [4].

Let $\delta p, \delta \rho$ and $\boldsymbol{q}(u, v, w)$ denote the perturbations in pressure $p$, density $\rho$ and velocity $\boldsymbol{U}(U(z), 0,0)$, respectively. Let the discontinuity in density occur at $z=z_{s}$, which after perturbation becomes

$$
z_{s}+\delta z_{s}(x, y, t)
$$

on account of Eq.(2.1), the discontinuity in the normal stresses required for equilibrium is

$$
T_{s}\left(\frac{\partial^{2}}{\partial x^{2}}+\frac{\partial^{2}}{\partial y^{2}}\right) \partial z_{s}
$$

Hence, the linearized perturbed equations of motion and continuity of the Rivlin-Ericksen elasticoviscous fluid in a porous medium (Rivlin and Ericksen [2], Sharma and Spanos [5]) are

$$
\begin{aligned}
& \frac{\rho}{\epsilon}\left[\frac{\partial \boldsymbol{q}}{\partial t}+\frac{1}{\epsilon}(\boldsymbol{U} \cdot \nabla) \boldsymbol{q}+\frac{1}{\epsilon}(\boldsymbol{q} \cdot \nabla) U(z) \hat{i}\right]= \\
& =-\nabla \delta p+\boldsymbol{g} \delta \rho-\frac{\rho}{k_{l}}\left(\mathrm{v}+\mathrm{v}^{\prime} \frac{\partial}{\partial t}\right) \boldsymbol{q}+\boldsymbol{n}_{S}\left[T_{S}\left(\frac{\partial^{2}}{\partial x^{2}}+\frac{\partial^{2}}{\partial y^{2}}\right) \delta z_{s}\right] \delta\left(z-z_{s}\right), \\
& \nabla \cdot \boldsymbol{q}=0
\end{aligned}
$$

where $\boldsymbol{n}_{s}$ denotes the normal to the macroscopic interface. $\in, k_{l}$, and $v^{\prime}$ stand for medium porosity, medium permeability, kinematic viscosity and kinematic viscoelasticity, respectively. Here, $\boldsymbol{g}(=0,0,-g)$ denotes the acceleration due to gravity and $\delta\left(z-z_{s}\right)$ stands for the Dirac delta function.

Since the density of a particle moving with the fluid remains unchanged, we have 


$$
\left[\in \frac{\partial}{\partial t}+\boldsymbol{U} \cdot \nabla\right] \delta \rho=-w \frac{d \rho}{d z}
$$

In Eq.(2.4), $\delta z_{s}$ can be expressed in terms of the normal component of the velocity $w_{s}$ at $z_{s}$ since

$$
\in \frac{d}{d t} \delta z_{s}=w\left(z_{s}\right)=w_{s}
$$

i.e., $\quad\left(\in \frac{\partial}{\partial t}+U_{s} \frac{\partial}{\partial x}\right) \delta z_{s}=w_{s}$

where the subscript " $s$ " indicates the value of the quantity at $z=z_{s}$. of the form

Analyzing the disturbances into normal modes, we seek solutions whose dependence on $x, y$ and $t$ is

$$
\exp \left[i\left(k_{x} x+k_{y} y+n t\right)\right]
$$

where $k_{x}, k_{y}$ are horizontal wave numbers, $k=\left(k_{x}^{2}+k_{y}^{2}\right)^{1 / 2}$ is the resultant wave number and $n$ is a complex constant.

Substituting for $\delta \rho, \delta z_{s}$ in Eq.(2.4) with the help of Eqs (2.5)-(2.8), we obtain

$$
\begin{aligned}
& {\left[\frac{i \rho}{\epsilon^{2}}\left(\in n+k_{x} U\right)+\frac{\rho}{k_{l}}\left(v+i n v^{\prime}\right)\right] \boldsymbol{q}+\frac{\rho}{\epsilon^{2}} w(D U) \hat{i}=} \\
& =-\nabla \delta p+i \boldsymbol{g} \frac{(D \rho)}{\left(\in n+k_{x} U\right)} w+i k^{2} T_{s}\left(\frac{w}{\epsilon n+k_{x} U}\right) \delta\left(z-z_{s}\right)
\end{aligned}
$$

where $\hat{i}$ is the unit vector in the $x$-direction and $D=\frac{d}{d z}$. obtain

Writing the three component Eqs of (2.9) and eliminating $u, v$ and $\delta p$ with the help of Eq.(2.5), we

$$
\begin{aligned}
& D\left[\left\{\frac{i \rho}{\epsilon^{2}}\left(\in n+k_{x} U\right)+\frac{\rho}{k_{l}}\left(v+i n v^{\prime}\right)\right\} D w-\frac{i k_{x} \rho}{\epsilon^{2}}(D U) w\right]+ \\
& -k^{2}\left[\frac{i \rho}{\epsilon^{2}}\left(\in n+k_{x} U\right)+\frac{\rho}{k_{l}}\left(v+i n v^{\prime}\right)\right] w=i g k^{2}\left[(D \rho)-\frac{k^{2}}{g} T_{s} \delta\left(z-z_{s}\right)\right] \frac{w}{\in n+k_{x} U} .
\end{aligned}
$$

\section{Two uniform streaming fluids separated by a horizontal boundary}

Here we consider the case when two superposed streaming fluids of uniform densities $\rho_{1}$ and $\rho_{2}$, uniform viscosities $\mu_{1}$ and $\mu_{2}$ and uniform viscoelasticities $\mu_{1}^{\prime}$ and $\mu_{2}^{\prime}$ are separated by a horizontal boundary at $z=0$. The subscripts 1 and 2 distinguish the lower and the upper fluids, respectively. 
The density $\rho_{z}$ of the upper fluid is taken to be less that the density $\rho_{l}$ of the lower fluid so that, in the absence of streaming, the configuration is stable and the porous medium throughout is assumed to be homogeneous and isotropic. Then in each of the two regions of constant $\rho, \mu, \mu^{\prime}$ and $U$; Eq.(2.10) reduces to

$$
\left(D^{2}-k^{2}\right) w=0 .
$$

The boundary conditions to be satisfied here are:

(a) Since $U$ is discontinuous at $z=0$, the uniqueness of normal displacement of any point on the interface according to Eq.(2.9) implies that

$$
\frac{w}{\in n+k_{x} U}
$$

must be continuous at an interface

(b) Integrating (2.10) between $z_{0^{-}} \eta$ and $z_{0^{+}}+\eta$ and passing to the limit $\eta=0$, we obtain, in view of Eq.(3.2), the jump condition

$$
\begin{aligned}
& \Delta_{0}\left[\left\{\frac{i \rho}{\epsilon^{2}}\left(\in n+k_{x} U\right)+\frac{\rho}{k_{l}}\left(v+i n v^{\prime}\right)\right\} D w-\frac{i k_{x} \rho}{\epsilon^{2}}(D U) w\right]= \\
& =i g k^{2}\left[\Delta_{0}(\rho)-\frac{k^{2} T_{S}}{g}\right]\left(\frac{w}{\epsilon n+k_{x} U}\right)_{0}, \quad(\text { for } z=0),
\end{aligned}
$$

while the equation valid everywhere else $(z \neq 0)$ is

$$
\begin{aligned}
& D\left[\left\{\frac{i \rho}{\epsilon^{2}}\left(\in n+k_{x} U\right)+\frac{\rho}{k_{l}}\left(v+i n v^{\prime}\right)\right\} D w-\frac{i k_{x} \rho}{\epsilon^{2}}(D U) w\right]+ \\
& -k^{2}\left[\frac{i \rho}{\epsilon^{2}}\left(\in n+k_{x} U\right)+\frac{\rho}{k_{l}}\left(v+i n v^{\prime}\right)\right] w=i g k^{2}\left[D \rho-\frac{k^{2}}{g} T_{s} \delta\left(z-z_{s}\right)\right]\left(\frac{w}{\in n+k_{x} U}\right) .
\end{aligned}
$$

Here $\Delta_{0}(f)=f\left(z_{0}+0\right)-f\left(z_{0}-0\right)$ is the jump which a quantity experiences at the interface $z=z_{0}$; and the subscript 0 distinguishes the value a quantity, known to be continuous at an interface, takes at the interface $z=z_{0}$.

The general solution of Eq.(3.1) is a linear combination of the integrals $e^{+k z}$ and $e^{-k z}$. Since $\left(\frac{w}{\in n+k_{x} U}\right)$ must be continuous on the surface $z=0$ and $w$ cannot increase exponentially on either side of the interface, the solutions appropriate for two regions are

$$
\begin{aligned}
& w_{1}=A\left(\in n+k_{x} U_{1}\right) e^{+k_{z}}, \quad(z<0), \\
& w_{2}=A\left(\in n+k_{x} U_{2}\right) e^{-k_{z}}, \quad(z>0) .
\end{aligned}
$$

Applying the boundary condition (3.3) to the solutions (3.5) and (3.6), we obtain the dispersion relation 


$$
\begin{aligned}
& {\left[1+\frac{\epsilon}{k_{1}}\left(\alpha_{1} v_{1}^{\prime}+\alpha_{2} v_{2}^{\prime}\right)\right] n^{2}+\left[\frac{2 k_{x}}{\epsilon}\left(\alpha_{1} U_{1}+\alpha_{2} U_{2}\right)+\frac{k_{x}}{k_{1}}\left(\alpha_{1} v_{1}^{\prime} U_{1}+\alpha_{2} v_{2}^{\prime} U_{2}\right)+\right.} \\
& \left.-\frac{i \in}{k_{1}}\left(\alpha_{1} v_{1}+\alpha_{2} v_{2}\right)\right] n+\left[\frac{k_{x}^{2}}{\epsilon^{2}}\left(\alpha_{1} U_{1}^{2}+\alpha_{2} U_{2}^{2}\right)-\frac{i k_{x}}{k_{1}}\left(\alpha_{1} v_{l} U_{1}+\alpha_{2} v_{2} U_{2}\right)+\right. \\
& \left.-g k\left\{\left(\alpha_{1}-\alpha_{2}\right)+\frac{k^{2} T_{s}}{g\left(\rho_{1}+\rho_{2}\right)}\right\}\right]=0,
\end{aligned}
$$

where $\quad \alpha_{1,2}=\frac{\rho_{1,2}}{\rho_{1}+\rho_{2}}, \quad v_{1,2}=\frac{\mu_{1,2}}{\rho_{1,2}}, \quad v_{1,2}^{\prime}=\frac{\mu_{1,2}^{\prime}}{\rho_{1,2}}$,

$v_{1}\left(=\frac{\mu_{1}}{\rho_{1}}\right), v_{1}^{\prime}\left(=\frac{\mu_{1}^{\prime}}{\rho_{1}}\right) ; v_{2}\left(=\frac{\mu_{2}}{\rho_{2}}\right)$, and $v_{2}^{\prime}\left(=\frac{\mu_{2}^{\prime}}{\rho_{2}}\right) ;$ are kinematic viscosities and kinematic viscoelasticity of lower and upper fluids, respectively.

Equation (3.7) yields

$$
\begin{aligned}
& \text { in }=-\frac{1}{2}\left(1+\frac{\epsilon}{k_{1}} \overline{\alpha_{1} v_{1}^{\prime}+\alpha_{2} v_{2}^{\prime}}\right)^{-1}\left[\frac{\epsilon}{k_{1}}\left(\alpha_{1} v_{1}+\alpha_{2} v_{2}\right)+\frac{2 i k_{x}}{\epsilon}\left(\alpha_{1} U_{1}+\alpha_{2} U_{2}\right)+\right. \\
& \left.+\frac{i k_{x}}{k_{1}}\left(\alpha_{1} v_{1}^{\prime} U_{1}+\alpha_{2} v_{2}^{\prime} U_{2}\right)\right] \pm \frac{1}{2}\left(1+\frac{\epsilon}{k_{1}} \overline{\alpha_{1} v_{1}^{\prime}+\alpha_{2} v_{2}^{\prime}}\right)^{-1}\left\{\left[\frac{\epsilon}{k_{1}}\left(\alpha_{1} v_{1}+\alpha_{2} v_{2}\right)\right]^{2}+\right. \\
& -\frac{4 i k_{x} \alpha_{1} \alpha_{2}}{k_{1}}\left(v_{1}-v_{2}\right)\left(U_{1}-U_{2}\right)+\frac{4 k_{x}^{2} \alpha_{1} \alpha_{2}}{\epsilon k_{1}}\left(v_{2}^{\prime} U_{1}-v_{l}^{\prime} U_{2}\right)\left(U_{1}-U_{2}\right)+ \\
& -\frac{2 i \in k_{x}}{k_{1}^{2}}\left[\left(\alpha_{1}^{2} v_{1} v_{1}^{\prime} U_{1}+\alpha_{2}^{2} v_{2} v_{2}^{\prime} U_{2}\right)+\alpha_{1} \alpha_{2}\left(v_{1} v_{2}^{\prime} U_{1}+v_{1}^{\prime} v_{2} U_{2}\right)+\right. \\
& +\alpha_{1} \alpha_{2}\left(U_{1}-U_{2}\right)\left(v_{1} v_{2}^{\prime}-v_{1}^{\prime} v_{2}\right)+\left[\frac{k_{x}}{k_{1}}\left(\alpha_{1} v_{1}^{\prime} U_{1}+\alpha_{2} v_{2}^{\prime} U_{2}\right)\right]^{2}+\frac{4 \alpha_{1} \alpha_{2} k_{x}^{2}}{\epsilon^{2}}\left(U_{1}-U_{2}\right)^{2}+ \\
& \left.-4 g k\left[\left(\alpha_{1}-\alpha_{2}\right)+\frac{k^{2} T_{s}}{g\left(\rho_{1}+\rho_{2}\right)}\right]\left[1+\frac{\epsilon}{k_{1}}\left(\alpha_{1} v_{1}^{\prime}+\alpha_{2} v_{2}^{\prime}\right)\right]\right\}^{1 / 2} \cdot
\end{aligned}
$$

\section{A. Absence of 'effective interfacial tension'}

In the absence of the 'effective interfacial tension', some cases of interest are now considered.

(a) when $k_{x}=0$, Eq.(3.8) yields

$$
\begin{aligned}
& \text { in }=\frac{1}{2\left\{1+\frac{\epsilon}{k_{l}}\left(\alpha_{1} v_{l}^{\prime}+\alpha_{2} v_{2}^{\prime}\right)\right\}}\left[-\frac{\epsilon}{k_{l}}\left(\alpha_{1} v_{1}+\alpha_{2} v_{2}\right) \pm\left\{\left[\frac{\epsilon}{k_{l}}\left(\alpha_{1} v_{l}+\alpha_{2} v_{2}\right)\right]^{2}+\right.\right. \\
& \left.\left.+4 g k\left(\alpha_{2}-\alpha_{1}\right)\left[1+\frac{\epsilon}{k_{l}}\left(\alpha_{1} v_{l}^{\prime}+\alpha_{2} v_{2}^{\prime}\right)\right]\right\}^{1 / 2}\right] .
\end{aligned}
$$


Here we assume kinematic viscosities $v_{1}, v_{2}$ and kinematic viscoelasticity $v_{1}^{\prime}, v_{2}^{\prime}$ of the two fluids to be equal, i.e., $v_{1}=v_{2}=v, v_{1}^{\prime}=v_{2}^{\prime}=v^{\prime}$. However, any of the essential features of the problem are not obscured by this simplifying assumption. Equation (3.9) then becomes

$$
\text { in }=\frac{1}{2\left(1+\frac{\in v^{\prime}}{k_{1}}\right)}\left[-\frac{\in v}{k_{l}} \pm\left[\left(\frac{\in v}{k_{l}}\right)^{2}+4 g k\left(\alpha_{2}-\alpha_{1}\right)\left\{1+\frac{\in v^{\prime}}{k_{l}}\right\}\right]^{1 / 2}\right] \text {. }
$$

(i) Unstable Case. For the potentially unstable configuration $\left(\rho_{2}>\rho_{l}\right)$, it is evident from Eq.(3.9) that one of the values of "in" is positive which means that the perturbations grow with time and so the system is unstable.

(ii) Stable Case. For the potentially stable configuration $\left(\rho_{2}<\rho_{l}\right)$, Eq.(3.10) yields that both the values of "in" are real, negative or complex conjugates with negative real parts implying thereby stability of the system.

It is interesting to note from the above that for the special case when perturbations in the direction of streaming are ignored $\left(k_{x}=0\right)$, the system is unstable for a potentially unstable configuration and stable for a potentially stable configuration and does not depend upon kinematic viscoelasticity, medium porosity and medium permeability. This is in contrast to the non-streaming (here $k_{x}=0$ ) Walters viscoelastic fluids $\mathrm{B}^{\prime}$ (Sharma et al., [6]) where the system can be stable or unstable depending upon whether the kinematic viscoelasticity is less than or greater than the medium permeability divided by medium porosity.

It is also clear from Eq.(3.9), that for the special case when perturbations in the direction of streaming are ignored $\left(k_{x}=0\right)$, the perturbations transverse to the direction of streaming $\left(k_{y} \neq 0\right)$, are unaffected by the presence of streaming.

(b) In every other direction, instability occurs when

$$
\frac{\alpha_{1} \alpha_{2} k_{x}^{2}}{\epsilon^{2}}\left(U_{1}-U_{2}\right)^{2}\left(1+\frac{\in v^{\prime}}{k_{1}}\right)>g k\left(\alpha_{1}-\alpha_{2}\right)\left(1+\frac{\in v^{\prime}}{k_{l}}\right) .
$$

The kinematic viscosities $v_{1}$ and $v_{2}$ and the kinematic viscoelasticities $v_{1}^{\prime}$ and $v_{2}^{\prime}$, of two fluids here are assumed to be equal (let $v_{1}=v_{2}=v, v_{1}^{\prime}=v_{2}^{\prime}=v^{\prime}$ ) but this simplifying assumption does not obscure any of the essential features of the problem.

Thus for a given difference in velocity $\left(U_{I^{-}} U_{2}\right)$ and for a given direction of the wave vector $\boldsymbol{k}$, instability occurs for all wave numbers

$$
k>\left[\frac{g \epsilon^{2}\left(\alpha_{1}-\alpha_{2}\right)}{\alpha_{1} \alpha_{2}\left(U_{1}-U_{2}\right)^{2} \cos ^{2} \theta}\right]
$$

where $\theta$ is the angle between the direction $\boldsymbol{k}\left(k_{x}, k_{y}, 0\right)$ and $\boldsymbol{U}(U, 0,0)$, i.e., $k_{x}=k \cos \theta$. Hence, for a given velocity difference $\left(U_{1}-U_{2}\right)$, instability occurs for the least wave number when $\boldsymbol{k}$ is in the directions of $\boldsymbol{U}$ and this minimum wave number; $k_{\min }$, is given by

$$
k_{\min }=\frac{g \in^{2}\left(\alpha_{1}-\alpha_{2}\right)}{\alpha_{1} \alpha_{2}\left(U_{1}-U_{2}\right)^{2}} .
$$

For $k>k_{\min }$, the system is unstable. 


\section{B. Presence of 'effective interfacial tension'} stability if

In the presence of the 'effective interfacial tension' in accordance with Eq.(3.8), there will be

$$
\left(1+\frac{\in v^{\prime}}{k_{1}}\right) \frac{k^{2} \alpha_{1} \alpha_{2}\left(U_{1}-U_{2}\right)^{2}}{\epsilon^{2}}<g k\left(1+\frac{\in v^{\prime}}{k_{1}}\right)\left[\left(\alpha_{1}-\alpha_{2}\right)+\frac{k^{2} T_{s}}{g\left(\rho_{1}+\rho_{2}\right)}\right] .
$$

Here we have set $k_{x}=k$ as these are the disturbances which are most sensitive to the KelvinHelmholtz instability, Eq.(3.4) as below

$$
\frac{\alpha_{1} \alpha_{2}\left(U_{1}-U_{2}\right)^{2}}{\epsilon^{2}}<g\left[\frac{\left(\alpha_{1}-\alpha_{2}\right)}{k}+\frac{k T_{s}}{g\left(\rho_{1}+\rho_{2}\right)}\right] \text {. }
$$

The R.H.S of this inequality has a minimum when

$$
\frac{d}{d k}(R H S)=0
$$

i.e., when $\quad-\frac{\left(\alpha_{1}-\alpha_{2}\right)}{k^{2}}+\frac{T_{s}}{g\left(\rho_{1}+\rho_{2}\right)}=0$,

or when $\quad \frac{\left(\alpha_{1}-\alpha_{2}\right)}{k^{2}}=\frac{T_{s}}{g\left(\rho_{1}+\rho_{2}\right)}$.

If we denote the value of $k$, given by Eq.(3.16) by $k^{*}$, there will be stability if

$$
\begin{aligned}
& \left(U_{1}-U_{2}\right)^{2}<\frac{g \epsilon^{2}}{\alpha_{1} \alpha_{2}}\left[\frac{\left(\alpha_{1}-\alpha_{2}\right)}{k^{*}}+\frac{\left(\alpha_{1}-\alpha_{2}\right)}{k^{*}}\right], \\
& \quad\left(U_{1}-U_{2}\right)^{2}<\frac{2 g \epsilon^{2}}{k^{*}} \frac{\alpha_{1}-\alpha_{2}}{\alpha_{1} \alpha_{2}} .
\end{aligned}
$$

Inserting the value of $k^{*}$ in accordance with Eq.(3.16)

$$
\text { i.e., } \quad k^{*}=\sqrt{\frac{g\left(\rho_{1}-\rho_{2}\right)}{T}},
$$

we conclude that the 'effective interfacial tension' will suppress the Kelvin-Helmholtz instability if

$$
\begin{aligned}
& \left(U_{1}-U_{2}\right)^{2}<2 g \in^{2} \frac{\alpha_{1}-\alpha_{2}}{\alpha_{1} \alpha_{2}} \sqrt{\frac{T_{s}}{g\left(\rho_{1}-\rho_{2}\right)}}, \\
& \text { i.e., if } \quad\left(U_{1}-U_{2}\right)^{2}<\frac{2 g \epsilon^{2}}{\alpha_{1} \alpha_{2}} \sqrt{\frac{T_{s} g\left(\alpha_{1}-\alpha_{2}\right)}{\left(\rho_{1}+\rho_{2}\right)}} .
\end{aligned}
$$


The streaming of the Rivlin-Ericksen elastico-viscous fluids in a porous medium has the same result Eq.(3.18) as that for the streaming of Newtonian fluids in a porous medium (Sharma and Spanos [5], inequality (34), p.1394). For a non-porous medium $\left(\in \rightarrow 1, k_{1} \rightarrow \infty\right)$, Eq.(3.18) yields the result due to Kelvin (cf. Chandrasekhar [1], inequality (40), p.486), implying thereby that the 'effective interfacial tension' completely suppresses the Kelvin-Helmholtz instability for small wavelengths.

The 'effective interfacial tension' is able to suppress this Kelvin-Helmholtz instability for small wavelength perturbations. The medium porosity reduces the stability range given in terms of a difference in streaming velocities.

\section{Conclusions}

A detailed account of stability of superposed Newtonian fluids, under varying assumptions of hydrodynamics and hydromagnetics, was given by Chandrasekhar [1]. With the growing importance of nonNewtonian fluids in chemical engineering, modern technology and industry, the investigations on such fluids are desirable. The Rivlin-Ericksen fluid is one such important non-Newtonian (visco-elastic) fluid. When the fluid permeates a porous material, the gross effect is represented by Darcy's law. As a result of this macroscopic law, the usual viscous term in the equation of Rivlin-Ericksen fluid motion is replaced by the resistance term $\left[-\frac{1}{k_{l}}\left(\mu+\mu^{\prime} \frac{\partial}{\partial t}\right) \boldsymbol{q}\right]$.

Visco-elastic fluids may have different effects as compared to Newtonian fluids, on the stability problems. For example, the effect of a uniform rotation on the thermal instability of a Maxwellian viscoelastic fluid is destabilizing, whereas the uniform rotation has a stabilizing effect on the thermal instability of a Newtonian fluid. Here the instability of two uniform, superposed and streaming Rivlin-Ericksen elasticoviscous fluids flow through porous media, including the 'effective interfacial tension', is considered. In the absence of the 'effective interfacial tension', the stability of the system for a potentially stable configuration does not depend upon kinematic visco-elasticity, medium porosity and medium permeability, whereas the presence of the 'effective interfacial tension' completely suppresses the Kelvin-Helmholtz instability for small wavelengths. The medium porosity reduces the stability range given in terms of a difference in streaming velocities.

For the potentially unstable configuration the perturbations grow with time in the absence of the 'effective interfacial tension'. The streaming of a Rivlin-Ericksen elastico-viscous fluid in a porous medium gives the same result as that for the streaming of Newtonian fluids in a porous medium.

\section{Nomenclature}

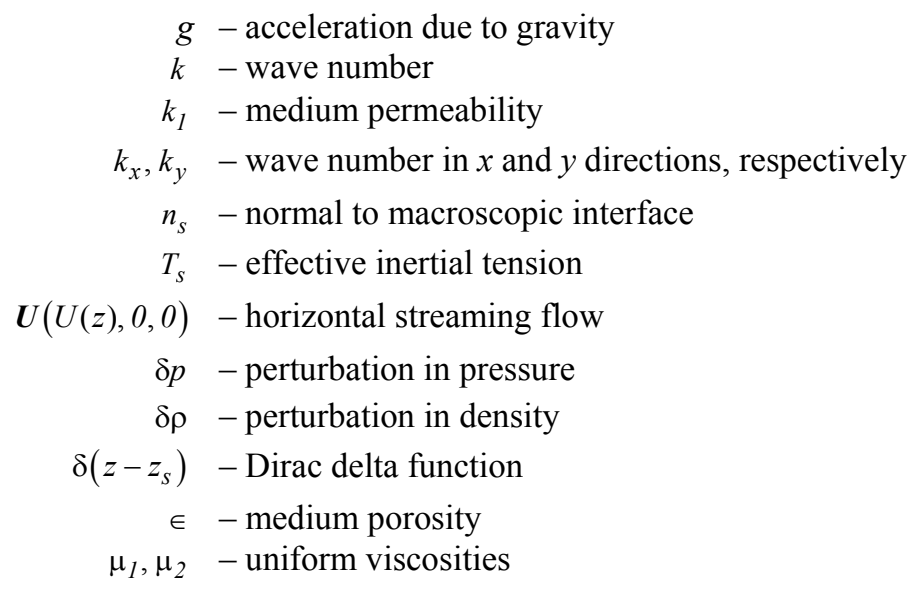




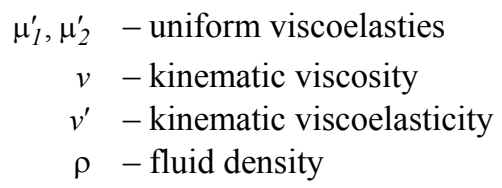

\section{References}

[1] Chandrasekhar S. (1981): Hydrodynamic and Hydromagnetic Stability. - New York: Dover Publication, pp.1-704.

[2] Rivlin R.S. and Ericksen J.L. (1955): Stress-deformation relations for isotropic materials. - J. Rational Mech. and Analysis, vol.4, pp.323-425.

[3] Phillips O.M. (1991): Flow and reaction in permeable rocks. - Cambridge: Cambridge University Press, X+285.

[4] Chuoke R.L., Van Meurs P. and Van der Poel C. (1959): Instability of slow immiscible viscous liquid -liquid displacement in permeable media. - Trans. AIME 216, pp.188-194.

[5] Sharma R.C. and Spanos T.J.T. (1982): The instability of streaming fluids in a porous medium. - Canadian J. Phy., vol.60, No.10, pp.1391-1395.

[6] Sharma R.C., Kumar P. and Sharma S. (2002): Rayleigh-Taylor instability of Walter's B' elastic-viscous fluid through porous medium. - Int. J. Appl. Mech. Engng., vol.7, No.2, pp.433-444.

Received: March 18, 2014

Revised: September 10,2015 